\title{
EXÁMENES EN LÍNEA DE INTRODUCCIÓN A LA INFORMÁTICA EN EL PROCESO DE ADMISIÓN DE LA UNIVERSIDAD CATÓLICA DE SANTIAGO DE GUAYAQUIL
}

\author{
ONLINE EXAMS OF INTRODUCTION TO COMPUTER IN THE ADMISSION OF \\ UNIVERSIDAD CATÓLICA DE SANTIAGO DE GUAYAQUIL
}

\author{
YADIRA DEL ROCÍO BELLO TOMALÁ
}

Universidad Católica de Santiago de Guayaquil. yadira.bello@cu.ucsg.edu.ec

RESUMEN

Este trabajo responde a una investigación realizada en las facultades de Ciencias Médicas y Arquitectura de la Universidad Católica de Santiago de Guayaquil (UCSG). Tuvo por objetivo proponer la implementación de un sistema en línea para la toma de los exámenes de Introducción a la Informática en el proceso de admisión a la UCSG, tomando en consideración las exigencias orientadas a la excelencia educativa. Luego del análisis del entorno en que se desarrolla el examen de exoneración de Introducción a la Informática y el tipo de evaluación utilizada desde el año 2010 hasta la actualidad, se determinó implementar esta nueva metodología con la intención de demostrar su contribución a la mejora de la calidad educativa, en esta área del conocimiento. La interactividad que presenta este tipo de evaluación en el área de Informática a través de internet, admite el uso de las nuevas tecnologías de la información y las comunicaciones de manera inquebrantable, por lo tanto, el medio cumple un rol socializador. El estudio realizado para este tipo de investigación experimental, se establece en una reforma institucional considerada para el mejoramiento de la calidad académica en el área de la Informática.

PALABRAS CLAVE: sistema interactivo, sistema en línea, simuladores, interfaz, evaluación, evaluación en línea.
This work responds to a research conducted in the Faculty of MTRACT Sciences and Architecture, which aimed to propose the implementation of an online system for taking exams of Introduction to Computer in the process of admission to the Catholic University of Santiago Guayaquil, taking into consideration the demands oriented educational excellence. After analyzing the environment in which the exemption exam Introduction to Computers and the type of assessment used since 2010 to the present, was determined to implement this new methodology, intended to demonstrate the contribution that can offer develops improving the quality of education in this area of knowledge. The interactivity of this type of evaluation in the area of Information on the Internet, supports the use of technology unswervingly, therefore, the medium plays a socializing role. The study for this type of experimental research, establishing an institutional reform considered for improving the academic quality in the area of Information Technology.

KEYWORDS: interactive system, on line system, simulators, front end, evaluation, online evaluation. 
INTRODUCCIÓN

La Universidad Católica de Santiago de Guayaquil (UCSG) acoge en su proceso de admisión aproximadamente a 10.000 aspirantes que se distribuyen entre las diferentes unidades académicas. Este período tiene dos momentos: el examen y el curso para el ingreso. Cada carrera tiene su propia malla curricular con materias básicas, generales (Lenguaje y Comunicación, Introducción a la Informática, Inglés) y otras propias de la carrera. Las materias generales son interfacultades, lo que quiere decir que los contenidos son los mismos en todas las unidades académicas.

En el caso de que el postulante esté inscrito en el examen para el ingreso, la nota obtenida en Introducción a la Informática, representa el $10 \%$ del total para aprobar; por lo que si en las otras materias obtuviese el $80 \%$ del puntaje no es relevante el resultado de ésta.

En el curso para el ingreso, el postulante debe dar el examen de exoneración durante la primera semana, si obtuviese la nota igual o mayor a 7,00 aprueba la materia. En caso contrario el postulante tiene la oportunidad de aprobar la cátedra mediante un curso remedial de 24 horas.

Este estudio plantea una reforma para que la toma del examen de exoneración de Introducción a la Informática en cualquiera de las dos modalidades del proceso de admisión, sea práctico tanto en línea como en modo presencial. La propuesta supone la utilización de un software que permita hacer la simulación de los programas sujetos de estudio y que por lo tanto incluya los contenidos de la materia.

La investigación propone una innovación en la forma de evaluar la materia Introducción a la Informática. Los recursos que se necesitan están al alcance de toda la comunidad universitaria debido a que la institución cuenta con la infraestructura adecuada para esta nueva metodología.

Chiluiza, Fernández y Caicedo (2010) destacan que Internet ofrece un gran potencial a la educación; como ejemplo se pueden mencionar las consultas, la investigación y el intercambio de experiencias y conocimientos, la intercomunicación entre autoridades, docentes, padres de familia y estudiantes. Son situaciones que conllevan el desarrollo de muchas capacidades y fortalecen la comunicación entre los involucrados en el hecho educativo (p. 150).
El uso de internet y sus múltiples aplicaciones cada vez es mayor, convirtiéndose de esta manera en un medio indispensable para la educación en el mundo entero, así como también en uno de los medios tecnológicos de la comunicación más reconocidos. Los avances alcanzados en el área tecnológica informática y de telecomunicaciones han permitido que el ser humano se desenvuelva de una manera más eficiente.

Según Lara (2001), la evaluación asistida por el computador consiste en el empleo de esta herramienta en algún momento del proceso de evaluación, tales como: definición, análisis y puntuación de los trabajos y exámenes, análisis de los datos, almacenamiento, análisis e informe en la ejecución, análisis y transferencia de la información de la evaluación a través de la red (p. 6).

Es por esto que la UCSC propone los exámenes en línea como una medida metodológica, tomando como parte de las consideraciones: el tipo de preguntas, el resultado de las notas, la relación que existe entre el número de aspirantes inscritos en el proceso de admisión y el número de docentes aptos para la elaboración, ejecución y revisión del examen de exoneración.

La metodología propuesta debe estar estructurada de forma tal, que marque una secuencia de conocimientos y habilidades que aseguren al postulante y a la institución, la consolidación de conocimientos y habilidades asistida por una computadora.

Es por ello que, en apego a los avances tecnológicos y acorde a la realidad, este estudio ofrece a las autoridades, docentes y estudiantes, una nueva metodología para evaluar Introducción a la Informática y que al mismo tiempo pueda satisfacer los requerimientos de la Institución.

\section{OBJETIVO GENERAL}

Implementar un sistema en línea para los exámenes de Introducción a la Informática en el proceso de admisión de la UCSG.

\section{OBJETIVOS ESPECÍFICOS}

1. Analizar varios programas de aplicación que permitan la elaboración de exámenes en línea.

2. Diseñar modelos de exámenes en línea que permitan la verificación de los conocimientos.

3. Diseñar la propuesta metodológica para los exámenes en línea de Introducción a la Informática. 
METODOLOGÍA

Se establecieron dos criterios de selección muestral: de tipo probabilística para la selección de estudiantes y no probabilística para la selección de los docentes.

El diseño experimental está orientado a evaluar la forma de tomar el examen de Introducción a la Informática y en base a sus resultados, hacer la propuesta de una nueva metodología. Los métodos y técnicas de investigación utilizados fueron de carácter cuantitativo.

La recogida de información se realizó mediante encuestas a estudiantes y docentes. Los datos obtenidos fueron tabulados con el programa Excel, así como los gráficos estadísticos.

El posterior análisis permitió conocer el grado de satisfacción que tienen los estudiantes y docentes acerca del tipo de examen y metodología empleados en los nuevos modelos de exámenes en línea.

Para el diseño de los modelos de exámenes se consideraron los contenidos del programa de estudio de la materia. Su validación constó de dos etapas: la rendición del examen y la respuesta a una encuesta de satisfacción sobre el examen práctico en línea que rindieron.

En este proceso de validación participaron estudiantes del primer ciclo de la carrera de Arquitectura y los del segundo ciclo de la carrera de Medicina, quienes aprobaron el examen de admisión 2014. Se tomó en cuenta esta población para la simulación de la prueba evaluativa práctica en línea, porque en el examen de admisión, la materia introducción a la Informática estuvo incorporada en el paquete junto con las demás materias que también forman parte del proceso de admisión.

De la misma manera participaron docentes que dictan la cátedra Introducción a la Informática en la UCSG. La lista de docentes que dictan esta materia fue entregada por Recursos Humanos de la institución. Cada estudiante o docente partícipe del estudio de campo realizó uno de los tres modelos de exámenes. Al finalizar la prueba tuvieron la oportunidad de revisar las preguntas ya contestadas, de imprimir el examen o de finalizarlo. Una vez que concluyeron la evaluación pudieron ver las respuestas contestadas erróneamente o ver la nota obtenida según lo contestado.

El diseño de estos modelos de exámenes en línea se realizó en la plataforma GMETRIX (analizada en la primera fase del proyecto de investigación), tomando en consideración los contenidos del programa de estudio de la materia, que la Comisión de Informática aprobó el 21 de septiembre de 2012 y el resultado que se obtuvo de una encuesta realizada sobre el nivel de satisfacción de los estudiantes del período de admisión 2014 de la carrera de Medicina, con respecto al examen teórico que rindieron.

Cada examen consta de diez preguntas, la calificación es sobre 100 y se definió como puntaje mínimo de aprobación 700 que equivale a 7,00 como está definido en la UCSG. La nueva modalidad de exámenes evalúa los resultados de aprendizajes detallados en el microcurrículo de la materia, aplica herramientas para la presentación de diapositivas, para el procesamiento de texto a un nivel básico y utiliza de forma adecuada el internet y el correo electrónico.

\section{RESULTADOS}

Los porcentajes obtenidos en cada pregunta de las encuestas realizadas a los estudiantes de las carreras de Arquitectura y Medicina, destacan la necesidad de mejorar el sistema de evaluación de la materia Introducción a la Informática en el proceso de admisión de la UCSG. Estos resultados tienen que ver con el nivel de satisfacción del examen en línea aplicados en la nueva metodología que se propone.

1. El porcentaje que indica estar "muy satisfecho" por el tipo del examen supera el $85 \%$.

2. El $96 \%$ de estudiantes está "muy satisfecho" con el tipo de preguntas y su nivel de dificultad.

3. Con respecto a las expectativas que tenían del examen, aproximadamente el 90\% indicaron estar "muy satisfecho".

4. El $98 \%$ indican que está "muy satisfecho" con la herramienta utilizada para la toma del examen.

5. El $80 \%$ de los estudiantes está "muy satisfechos" con el tiempo de duración de la prueba.

6. El $98 \%$ de estudiantes está "totalmente de acuerdo" con que el examen de Introducción a la Informática sea práctico y con la importancia que tienen las herramientas informáticas en su carrera profesional.

De la encuesta realizada a los docentes de la UCSG, se pueden resumir los siguientes aspectos:

El $100 \%$ de los docentes que validaron la prueba y fueron encuestados, están "muy sa- 
tisfechos" con el examen práctico en línea que rindieron, el tipo de preguntas contenidas en el examen, la herramienta utilizada para la toma del examen, el nivel de dificultad de las preguntas y el tiempo de duración del examen.

El $100 \%$ de los docentes que validaron la prueba contestaron en la encuesta que están "totalmente de acuerdo" con que el examen de la materia Introducción a la Informática debe ser práctico, y que este es el tipo de examen idóneo para exonerar esta materia.

Sugerencias y opiniones de docentes a partir de las encuestas que les fueron suministradas:

1. Incrementar a 15 el número de preguntas y a 30 minutos el tiempo de duración del examen.

2. Ahorra el tiempo de estar revisando para cada estudiante el examen y permite obtener resultados inmediatos.

3. Se debe implementar lo más pronto posible.

4. Es una excelente herramienta.

5. Se debería aplicar también a Informática I.

6. Totalmente de acuerdo con este proyecto, se deben actualizar las herramientas de apoyo en tecnologías de la información y comunicación.

CONCLUSIONES

Introducción a la Informática se imparte en el proceso de admisión en todas las carreras de la UCSG. No es novedoso que los aspirantes tengan un conocimiento previo de las herramientas informáticas que se evalúan, lo que se trata de medir son las habilidades o competencias que tienen en el uso de estas aplicaciones. El examen que se toma en la actualidad es de tipo objetivo - teórico por lo que difícilmente puede cumplir con esta pretensión de medición.
En el área de Informática, el nivel de conocimiento con el que ingresan los aspirantes que aprueban el proceso de admisión es heterogéneo. Este depende de la modalidad del sistema de admisión.

Los resultados de las encuestas y la validación del nuevo tipo de examen demuestran el alto nivel de satisfacción con la nueva metodología que se pretende implementar para la toma del examen en línea de Introducción a la Informática. Más del $80 \%$ de los estudiantes y el $100 \%$ de los docentes que validaron la prueba piloto y fueron encuestados están totalmente de acuerdo con este cambio de metodología.

La elaboración de la evaluación en línea en la plataforma Cmetrix, da soporte a una aplicación tecnológica que tiene como pilares fundamentales a las ciencias informáticas y de la educación, tal como lo demuestran los resultados obtenidos en la metodología empleada en la recolección de información para esta fase del trabajo de investigación.

Finalmente se puede decir que esta nueva metodología aplicada en la toma de examen en línea de Introducción a la Informática es una alternativa que tendría la institución para dar un giro favorable en la forma de evaluar esta cátedra.

\section{REFERENCIAS BIBLIOGRÁFICAS}

Chiluiza García, K., Fernández Ronquillo, E., y Caicedo Alarcón, B. (2010). Introducción a las tecnologías de la información y la comunicación. Ecuador: Ministerio de Educación del Ecuador.

Lara, S. (2001). La evaluación formativa a través de Internet. Aplicaciones informáticas y experiencias prácticas (Educación). Navarra: Ediciones Universidad de Navarra, S.A. 